\title{
Principle of Internet of Intelligences and Development of its Core Technology
}

\author{
Chongfu Huang ${ }^{1,2,3}$ \\ 1. Key Laboratory of Environmental Change and Natural Disaster, Ministry of Education of China, Beijing Normal \\ University, Beijing 100875, China \\ 2. Academy of Disaster Reduction and Emergency Management, Faculty of Geographical Science, Beijing Normal \\ University, Beijing 100875, China \\ 3. Beijing Cazl Technology Service Co., Ltd., Beijing 100088, China \\ E-mail: hchongfu@bnu.edu.cn
}

Received 5 September 2017

Accepted 28 September 2017

\begin{abstract}
The network platform connecting multiple agents, which is embedded in the models to integrate individual wisdoms into a great wisdom, is called Internet of intelligences (IOI). The risk radar can be driven by IOI with mathematical models written by PHP language. A measure space of the seismic macro-anomalies group was set up in IOI based the consensus of the staffs participating earthquake monitoring and prediction. The core technology in IOI is information diffusion technology, and its intelligent development depends on the appearance of intelligent mathematics on earth. By means of the development of the factor space theory to construct IOI expressing knowledge and being cognizant of thinking, we should promote the research of "Internet + Risk Analysis" and promote the development of Web mathematics, and lay the foundation for intelligent mathematics. The IOI supported by Web mathematics is expected to decompose and dissolve wisdom ingredients in some factor spaces so that individual wisdoms are integrated to be a great wisdom.
\end{abstract}

Keywords: Internet of Intelligences; Risk; Risk Radar; Information Diffusion; Factor Space; Web Mathematics; Intelligent Mathematics

\section{智联网原理及其核心技术的发展}

\section{黄崇福 $1,2,3$}

1. 北京师范大学环境演变与自然灾害教育部重点实验室, 北京 100875, 中国

2. 北京师范大学地理科学学部减灾与应急管理研究院, 北京 100875 , 中国

3. 北京崇安智联科技服务有限责任公司，北京 100088 , 中国

\begin{abstract}
摘 要: 由互联网联结多个智能体, 并通过嵌入的模型实现集个体小智慧为群体大智慧的网络平台, 称为智 联网。用 PHP 语言编写数学模型所支持的智联网平台, 已经实现了驱动风险雷达和建立共识性地震宏观异常 度量空间。智联网的核心技术是信息扩散技术, 其智能化的发展, 依赖于地球上出现智能数学。通过发展因 素空间理论来构建表达知识和认知思维的智联网, 既能推动 “互联网+风险分析” 的研究, 还能促进网络数 学的形成, 为智能数学打下基础。网络数学支持的智联网, 有望用因素空间来分解、溶合智慧成分, 将小智 慧提升为大智慧。
\end{abstract}

关键词: 智联网; 风险; 风险雷达; 信息扩散; 因素空间; 网络数学; 智能数学

* 本项目受国家自然科学基金项目(No.41671502)的资助, 部分受北京崇安智联科技服务有限责任公司的资助。 


\section{1. 引言}

用有限的知识, 认识无限的世界, 是人类永恒 的追求。用不完备信息和有限的知识, 研究不确定 性极大的与不利事件有关的未来情景, 这就是风险 分析的工作。风险是一种扑塑迷离的现象, 风险分 析不是将数据放入模型中算一算的机械性工作, 而 是一项智能工作。随着互联网的广泛应用, 智联网 技术应运而生 ${ }^{[1]}$, 为集众人之所长, 生超级之智能, 解风险之万千, 提供了一个重要的渠道。同时, 相 关的研究, 将为互联网时代人工智能的研究提供一 个检验平台。

由互联网联结多个智能体, 并通过嵌入的模型 集个体小智慧为群体大智慧的网络平台, 称为智联 网。目前, 人们已经研制出 9 个智联网平台。

高考志愿填报智联网服务平台 ${ }^{[2]}$ 是首个研制成 功的智联网演示系统。考生以购买服务的方式, 通 过智联网获得信息和咨询, 并由数学模型处理后提 供决策参考, 有效规避填报失误的风险。台风灾害 风险分析智联网 ${ }^{[3]}$ 中出现了柔性知识捕获器, 以在温 州地区开展台风灾害水产养殖保险的可行性需求调 查为例, 对智联网平台的理念和模型进行了成功的 验证。智联网支撑的 “优秀论文” 评选系统 ${ }^{[4]}$ 能评出 共识性最高的优秀论文, 多次用于中国风险分析与 管理精英杯优秀论文的评选。内涝风险分析智联网 服务平台 ${ }^{[5]}$ 使用属性拼图技术对原始的经验信息进 行优化整合, 再通过 “雨强一水深” 模糊关系模型, 在线实现基于降雨强度, 估计易涝点积水深度, 进 而给出积水风险值。智联网驱动的风险雷达 ${ }^{[6]}$ 通过社 区居民的参与, 进行风险信息搜集、动态风险评估, 实现了风险事件的动态追踪。该项发明已由北京崇 安智联科技服务有限责任公司申请了名为 “一种社 区安全风险雷达智联网服务系统” 的发明专利。海 洋环境风险管理智联网平台 ${ }^{[7]}$ 通过去中心化信息收 集的技术, 完成了围填海造地项目对天津自然灾害 抵御能力的影响评价。地震宏观异常的智联网服务 平台 ${ }^{[8]}$ 将众多一线地震工作者对地震宏观异常群强 度的目视判断, 以模糊隶属函数的曲线方式输入, 在智联网中建立以模糊关系矩阵表达的共识性宏观 异常群测度空间, 有望为震前宏观异常辅助地震预 报提供帮助。风险时效性评价的智联网服务平台 ${ }^{[9]}$ 是一个因素藤智联网, 被用于对北京延庆区果树冰 雨灾害概率风险的时效性进行评价。风险沟通智联 网服务平台 ${ }^{[10]}$ 提供了洪水灾害风险沟通的便捷渠
道, 并尝试用于宁波市洪水灾害的风险管理。

上述这些智联网, 无论是其遵循的原理, 还是 集值统计意义上的信息扩散核心技术, 均是传统数 学模型; 加之缺少合适的商业模式, 目前还没有被 大规模地应用。如果能跳出传统数学模型的约束, 实现较高水平的智能化, 哪么, 既使不靠商业模式, 仅仅靠技术优势, 也会有更多的人依托智联网开展 风险分析的工作，使之成为 “互联网+风险分析” 的 主流。

本文将在第 2 节介绍智联网原理, 给出相关定 义和编程方式; 第 3 节介绍智联网中的核心技术 “信 息扩散”; 第 4 节提出了对网络数学的设想; 第 5 节 研究因素空间理论作为网络数学出发点的问题; 第 6 节探讨如何网络数学支持智联网, 第 7 节给出结论 和一些后续问题的讨论。

\section{2. 智联网原理}

人类社会经历了从农业阶段到工业阶段, 再到 信息化阶段三次巨大浪潮。目前, 人类正处于第三 次浪潮之中, 其特点是形形色色的互联网无处不在。 移动通信、物联网、战术数据链等大型的信息系统, 无一例外皆有互联网的烙印。然而, 今天的互联网 系统, 远非智能系统, 更多的是信息发布和交换系 统。

今天, 以深度学习为特征, 以大数据为背景的 人工智能被炒得沸沸扬扬。AlphaGo 横扫 60 位围棋 大师; Watson 制定癌症治疗方案, 都是标志性的事 件。一夜之间, 似乎人工智能已从虚无缥缙的幻想 成为了现实。如此这般, 人工智能完成风险分析的 工作, 应该更不在话下。然而, 事实并非如此, 这 些奇妙的人工智能, 本质上与工业革命时代的自动 化同出一辙, 并非智能化。正如日本发明的模糊技 术控制的洗衣机被冠以智能洗衣机之名, 但其实并 无智能, 只是自动化程度高一点而已。人类智能的 标志是能创造知识。只有能创造知识的机器智能, 才是真正的人工智能。计算机系统用某种技巧拼凑 出乐曲, 用某些模板几十秒内写出重大自然灾害事 件的新闻稿子, 已经被商业包装为 “人工智能” 产 品, 但其与真正的人工智能, 毫无关系。自动化意 义下的智能, 应该被称为 “灵巧”, 英文为 Smart; 能创造知识的智能, 实为 “智慧”, 才称得上英文的 Intelligence。

显然, 依靠人类智能进行的风险分析工作, 不 可能由 Smart 式的人工智能替代。依靠网络的支持, 
做大做强人类智能，或许是人工智能热潮中，提高 风险分析水平的一条重要渠道。建设这条渠道的工 具之一, 是智联网。

“智联网” (Internet of Intelligences) 是 在互联网的服务器上, 使用一系列数学模型, 利用 人机交互技术，实时处理来自众多利益相关者的信 息、经验和判断，对客观现象做出综合分析的网络 平台。

我们可以将智联网与物联网做一个比较, 说明 智联网的本质所在。物联网无需人的干预, 其实质 是利用射频自动识别技术，通过计算机互联网实现 物品的自动识别和信息的互联与共享。智联网须有 人参与, 其实质是用数学模型整合信息和经验, 将 个人判断的小智慧提升为群体大智慧, 生成智能产 品。物联网是一个信息管理系统, 智联网是一个智 慧加工和生产系统。

智联网原理由两大部分构成,一是形式化定义; 二是编程原理。

\section{1 智联网的形式化定义}

定义 1 . 具有观察、演绎、推理和解决问题能力的个 体称为智能体。

例如，正常的个人、决策支持系统、恒温调节 器、模糊洗衣机等, 均是智能体。

定义 2. 设 $A$ 是一个智能体集合, $N$ 是 $A$ 使用的一个 网络, $M$ 是处理 $A$ 所提供信息的模型, 三元体 $<A, N$, $M>$ 称为一个智联网, 记为 $\Phi$ 。

换言之, 一个由智能体、计算机网络和信息处 理器组成的系统, 称为一个智联网。

最简单的智联网可以用图 1 示之, 该系统由三 个智能体 $a_{1}, a_{2}, a_{3}$, 三台终端机 $c_{1}, c_{2}, c_{3}$, 一台服务 器 $S$ 和一个信息处理模型 $M$ 组成。它的拓扑结构见 图 2。智联网的工作原理见图 3。

互联网是一个动态混沌系统, 没有人知道每一 个节点何时会发生什么变化, 也不知这种变化对其 它节点会产生什么影响。智联网则是一个智力提升 系统, 只有当其数学模型 $M$ 能加工生产出智慧产品 时, 系统才有存在的意义。智联网具有动态自组织 性, 目标是形成一个高级智能体。互联网从混沌系 统走向自组织系统，必将是一次革命。

事实上，互联网应用模式的发展和人类大脑的 结构机理, 有惊人的重合。例如, 从电子邮件、电 子公告牌到博客、社会化网络，互联网的每一个应
用创新都能映射到人脑的功能结构中 ${ }^{[11]}$ 。人们预测, 互联网的应用模式将形成一个与人类大脑功能结构

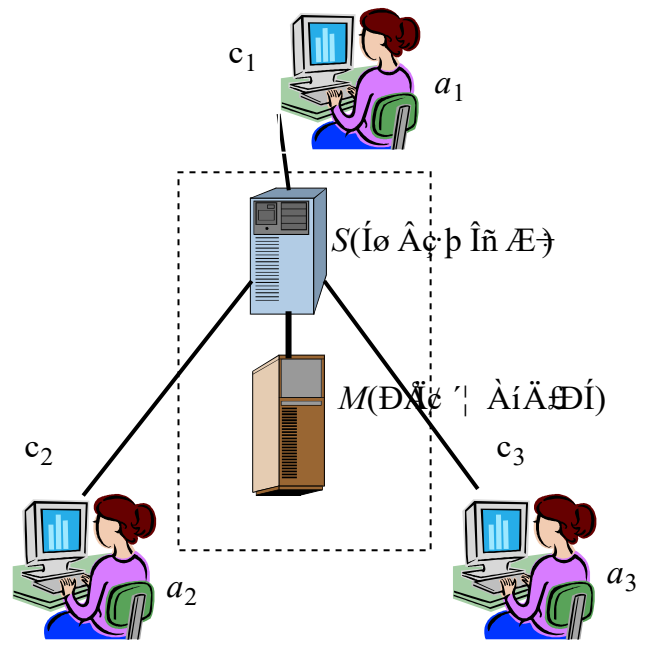

图 1. 最简单的智联网 ${ }^{[1]}$ 。图中的三台计算机形成网络, 三 个工作人员是三个智能体。

高度相似的网络虚拟结构, 互联网正朝着使人脑充 分互联的方向发展和进化, 全球脑呼之欲出 ${ }^{[12]}$ 。

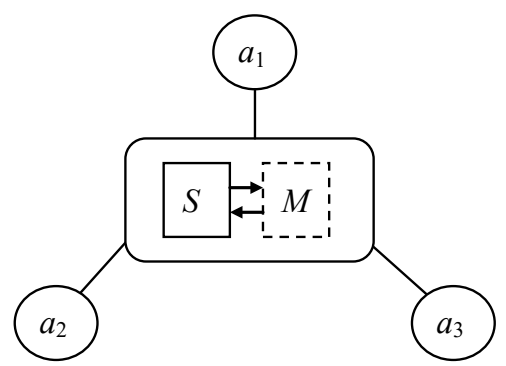

图 2. 一个简单智联网的拓扑结构 ${ }^{[13]}$, 由一个网络服务器 $S$, 一个数学模型 $M$ 和的三个智能体 $a_{1}, a_{2}, a_{3}$ 组成。

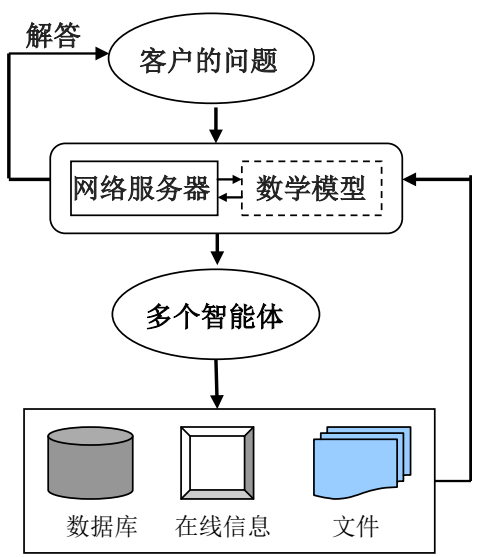

图 3. 智联网工作原理 ${ }^{[13]}$ 。智联网用数学模型处理来自智能 体的在线信息，在一些背景数据库的支持下，智联网为客 户解答问题。 


\section{2 智联网的编程原理}

智联网是一个计算机网络系统平台，用网络编 程语言来实现。编程原理是: 用 HTML 语言实现终 端界面, 用 MySQL 建设服务器上的数据库, 用 PHP 语言连接界面和数据库并编写数学模型。这一原理 如图 4 所示。

\subsubsection{HTML 语言}

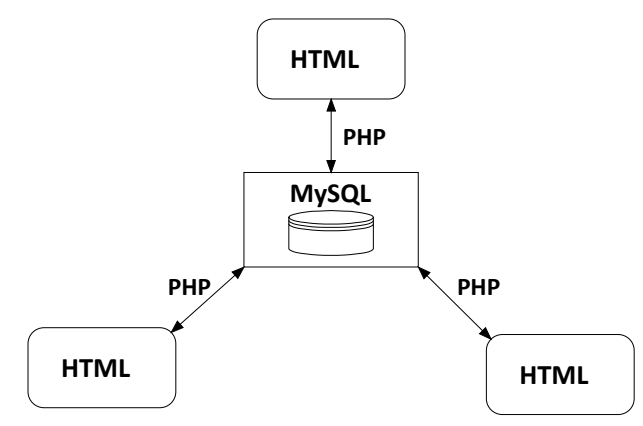

图 4. 智联网编程原理示意图。智联网平台由三个部分构 成: 终端界面、服务器上的数据库和连接界面和数据库并 能运行数学模型的网络程序。它们分别由 HTML 语言、 MySQL 数据库和 PHP 语言来实现。

智联网的终端界面, 由网页编写技术实现。 HTML（HyperText Mark-up Language）即超文本标 记语言, 是目前网络上应用最为广泛的网页编写语 言, 它提供了终端界面的编写技术。

HTML 文本是由 HTML 命令组成的描述性文 本, HTML 命令可以说明文字、图形、动画、声音、 表格、链接等。HTML 的结构包括头部 ( Head)、主 体 (Body) 两大部分, 其中头部描述浏览器所需的 信息, 而主体则包含所要说明的具体内容。

用记事本编写 HTML 语言的文件, 并以后缀名 “html” 存之, 用汶览器就可以打开所设计的终端界 面。为了方便开发终端界面, 人们研制了诸如 editplus、Dreamweaver 和 zend studio 等大量的开发 工具, 使 HTML 文件的编写, 更加方便。

\subsubsection{MySQL 数据库}

互联网本身就是一个大型的分布式超媒体信息 数据库, 但服务于智联网的数据库, 是一个能用互 联网访问的专用数据库。MySQL、Oracle 和 SQL Server 数据库都可以使用, 但 Oracle 过于庞大, SQL Server 的安全性较差, 在智联网中我们使用 MySQL。

MySQL 是一种开放源代码的关系型数据库管 理系统, 任何人都可以下载并根据个性化的需要对
其进行修改。通过数据库管理系统, 管理者可以轻 松地实现各种数据库对象的访问 (增、删、改、查 等操作), 并可以轻松地完成数据库的维护工作 (备 份、恢复、修复等操作)。

MySQL 数据库的管理者, 通过调用操作系统的 进程管理、内存管理、设备管理以及文件管理等服 务, 为数据库用户提供管理、控制数据库容器中各 种数据库对象、数据库文件的接口。

不掌握数据库管理系统的数据库用户，则无法 经由网络获取数据库文件中被管理者屏蔽了的具体 内容。

MySQL 的功能并不很强大, 但因为它的开源、 广泛传播, 是智联网首选的数据库。

\subsection{PHP 语言}

连接界面和数据库并能运行数学模型的网络程 序, 用 PHP 语言编写。PHP (Hypertext Preprocessor) 即超文本预处理器, 是一种通用开源脚本语言, 其 语法吸收了 C 语言、Java 和 Perl 的特点, 程序嵌入 到 HTML 文档中去执行。

PHP 脚本在服务器上执行, 然后向浏览器发送 回 HTML 结果。PHP 脚本可放置于 HTML 文档中 的任何位置, 通常以“<?php”开头, 以“? ”结尾。 在 PHP 中, 所有用户定义的函数、类和关键词（例 如 if、else、echo 等等) 都对大小写不敏感，而对 所有变量的大小写敏感。

参照最流行和使用最为广泛的能编排漂亮数学 公式的 LaTex 排版系统, PHP 对数学变量使用“\$” 进行标注。PHP 程序中的变量以 $\$$ 符号开头, 其后 是变量的名称。一个用一维信息分配方法进行概率 分布估计的 PHP 程序见图 5 。

\begin{tabular}{|c|}
\hline 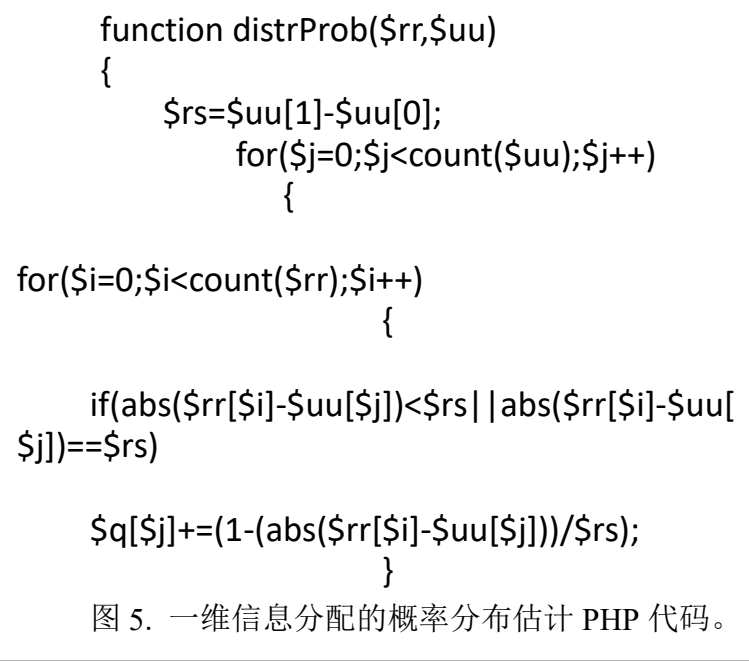 \\
\hline
\end{tabular}




\section{3. 智联网的核心技术: 信息扩散技术}

针对具体问题, 能拥有信息和经验参与智联网 解答问题的人员并不多。大多数情况下, 智联网是 在小样本条件下进行工作。这与欧盟第七框架计划 大型项目 iNTeg-Risk 推出的欧洲新兴风险雷达---风 险滴嗒器 ${ }^{[14]}$---大不相同。

风险滴嗒器也是一个计算机网络系统平台, 但 它是通过网络爬虫实时抓取互联网中的灾害报道并 计算出风险指数, 它还能通过推特(twitter)中某地对 一种传染病的讨论人数多少来评估此种传染病在该 地的风险。讨论的人数越多, 被认为风险水平越高。 风险滴嗒器追踪的是宏观风险, 使用的是互联网中 大量的信息。依赖网络大数据的风险滴嗒器, 监测 不了微观的风险。

例如, 对于一个社区的火灾风险, 只有发现了 火灾隐患的少数居民和相关管理人员, 才能提供有 价值的信息和有针对性的经验, 智联网中的数学模 型才能计算出该社区的风险。而社区外议论火灾风 险的人再多, 网络数据再丰富, 对该社区的实时风 险监测, 提供不了任何帮助。智联网驱动的风险雷 达, 是为某一区域专门研制的网络平台, 配备有该 区域的基础数据库, 只关注该区域的风险, 追踪的 是微观风险。由于大多数风险源显露时, 区域中往 往只有几个人发现, 为实时而快捷地追踪风险, 须 在只有少量信息的情况下做出风险评估。少量信息 进入评估模型, 我们就遇到了小样本问题。

智联网对问题解答的质量由微观、实时和快捷 来决定。这就要求智联网必须具备处理小样本的能 力。网络中的大数据, 只具有补充和改善基础数据 库的作用。

信息扩散原理保证了信息扩散技术能优化处理 小样本, 显著提高分析精度。信息扩散的基本思想 是把一个传统的数据样本点变成一个模糊集合。由 于信息扩散的目的是挖掘出尽可能多的有用信息, 以此提高系统识别的精度, 这种技术, 也被称为模 糊信息优化处理技术。最简单的扩散技术是线性信 息分配方法, 应用最广泛的扩散技术, 是正态扩散。

$$
\text { 令 } D=\left\{x_{1}, x_{2}, \ldots, x_{n}\right\} \text { 是一个给定的样本点集 }
$$

合, $U=\{u\}$ 是它的论域（定义域）。将 $x_{1}, x_{2}, \ldots, x_{n}$ 视为物理化学意义上的分子, 将 $U$ 视为分子扩散的 空间, 模仿分子扩散, 可以给出信息扩散方程, 得 到一个扩散函数:

$$
\mu(x, u)=\frac{1}{h \sqrt{2 \pi}} \exp \left[-\frac{(x-u)^{2}}{2 h^{2}}\right], \quad x \in D, u \in U .
$$

由于 $\mu(x, u)$ 表达的, 给定样本点 $x$ 沿论域点 $u$ 进行 扩散的量值分布, 正好与概率论中的正态分布相同, 所以 $\mu(x, u)$ 被称为正态扩散函数。其归一化的模糊 集由式（2）表达。

$$
\mu_{x}(u)=\exp \left[-\frac{(x-u)^{2}}{2 h^{2}}\right], \quad x \in D, u \in U .
$$

用式 (1) 和式 (2) 对样本点 $x$ 在 $U$ 上进行的 信息扩散处理, 都称为正态信息扩散。由于对模糊 集样本点进行统计分析时, 须进行归一化处理, 让 各个模糊集样本点具有相同的统计地位, 所以, 式 (1) 中的第一个因子不影响统计结果。通常提及的 正态信息扩散, 是指用式 (2) 进行的扩散。

对正态扩散的计算机仿真实验表明, 当每一个 信息接收点, 从邻近两个样本点扩散得到信息量, 等于或多于从其它样本点得到的信息量总和时, 扩 散估计的结果最好。这一现象被称为两点择近。根 据两点择近原则和样本点平均距离假设, 可以推导 出一个计算扩散系数的简单公式:

$$
h= \begin{cases}0.8146(b-a), & n=5 ; \\ 0.5690(b-a), & n=6 ; \\ 0.4560(b-a), & n=7 ; \\ 0.3860(b-a), & n=8 ; \\ 0.3362(b-a), & n=9 ; \\ 0.2986(b-a), & n=10 ; \\ 2.6851(b-a) /(n-1), & n \geq 11 .\end{cases}
$$

这里 $\quad b=\max _{1 \leq i \leq n}\left\{x_{i}\right\}, \quad a=\min _{1 \leq i \leq n}\left\{x_{i}\right\}$.

使用信息扩散方法, 不需要关于样本点之间内 在联系（诸如概率分布类型等）的任何人为假设, 从而推断出来的结论不会因人而异。

目前, 用于智联网的信息扩散技术, 是一种统 计样本的集值化技术, 旨在提高统计精度。这种技 术的作用域是概率空间, 而非物理空间。使用这种 技术，不需要任何空间性质的支持。

人们正在研究的 “空间不完备信息的扩散技 术”, 试图通过扩散函数与地理空间中自然属性和社 会经济属性的共振, 建立弥补地理单元数据缺失的 信息扩散技术, 使之比假设空间数据连续而插值的 数据更符合实际, 比诸如反距离移动平均法、反距 离移动表面法和普通克里格插值法等得到的数据都 更具可解释性。 


\section{4. 智联网与网络数学}

智联网的核心，不是商业模式，不是互联网技 术, 更不是数学模型的复杂度, 而是能否集小智慧 为大智慧。

举例来说，10 个刚出道的风险投资人，对一个 项目的风险评估, 各人都会有明显的考虑不到之处。 而一名成功的风险投资家 (例如, “股神”巴菲特), 会考虑得更加周到。是不是综合 10 个新手的评估结 果, 就能有所改进了呢? 当然不是, 众人看走眼的 事, 很多。在众人均看错的情况下, 无论用什么好 的传统数学模型去综合众人的观点, 结果也还是错。 如果有一个平台, 能帮助这 10 个新手协同工作, 评 估出与成功风险投资家一样的或差不多的结果, 这 个平台就具有了集小智慧为大智慧的功能。

作者认为, 目前的数学, 不是用来表述人类思 维活动的工具, 不是智能数学, 不能提供集小智慧 为大智慧的数学支持。使用传统数学模型的智联网, 只具有在线整合部分信息和经验, 快速做出较合理 判断的能力。社区安全风险雷达, 较好地发挥了现 有智联网的这一特长。

在思维科学领域, 人们最早关注的是计算思维 (Computational Thinking)。主流学派是把人脑设想 成一套复杂的开关网络, 努力发展数学模型来模拟 大脑思维。人工神经元网络, 是较为成功的模型之 一。受此启发, 上世纪末, 人们发展了大量的生物 计算模型, 自然计算 (Natural Computation) 概念应 运而生。以模糊逻辑为主的软计算, 有力地推动了 计算智能(Computational Intelligence)的发展。这些用 现有数学原理开发的数学模型, 既使模拟了人的某 种思维过程, 在认识问题和解决问题方面有所发展, 甚至在计算机上得以实现, 但仍是 Smart 意义下的 智能, 而非 Intelligence 意义下的智能, 因为这些 数学模型并不具备集小智慧为大智慧的潜能。

人们停留在 Smart 水平的根本的原因, 不是开 发数学模型的努力不够, 也不是计算机还不够发达。 根本的原因是现有的数学原理不支持集小智慧为大 智慧的数学模型的出现。

事实上, 人类从第一代数学进化到第二代数学 后, 就没有质的进步。本文将研究静态问题的数学 称为第一代数学; 研究动态问题的数学称为第二代 数学。只有出现第三代数学, 人类才具有研究智慧
问题的数学工具。本文将第三代数学称为智能数学。 当然, 并非文[15]认为的, 具有格结构的模糊数学, 就是智能数学。模糊数学是第二代数学中一个很小 的部分。

人们从简单的计数到美轮美负的代数系统, 并 坚韧不拔地在证明诸如哥德巴赫猜想等大量关于 “数” 的定律, 历经几千年, 构建了第一代数学; 从牛顿研究天体运动规律的需要, 到柯西简洁而严 格地证明了的牛顿-莱布尼茨微积分学, 再到充满争 议的主观-非主观概率论, 历经三百多年, 人们构建 了第二代数学。这两代数学, 组成了体系宏大的传 统数学, 严密而富有活力, 为现代科学和技术的发 展, 提供了重要的工具。但是, 到目前为止, 没有 一门数学学科是因为研究智慧的生成、发展和增强 而发展起来。人们已经满足于用传统数学的良性拓 展来研究天地万物, 也包括研究智能问题。

超越 Smart 的智能数学, 目前只能是一个泛泛 而谈的第三代数学, 实质性的探索的工作之一, 或 许可以从建立网络数学开始。

目前, 无论是 IMB 的“Watson”, 还是在线或非 在线的网页语义分析, 抑或是棋技超群的 Google 的 AlphaGo, 大凡用到数学, 无一不是以传统数学为基 础。没有一门数学学科是因为研究互联网中的各种 现象而发展起来的。

然而, 正如将传统数学的十进制数用于计算机 设计, 电路很难实现一样, 以传统数学为基础来研 究互联网中的各种现象, 注定效率低下。虽然十进 制表述的任何数学问题均可转化为用二进制表述, 成为计算机能读懂的问题, 但如果没有二进制, 没 有布尔代数, 就没有今天的计算机科学。同理, 没 有网络数学的出现, 基于传统数学的模型, 不可能 支持高效的智联网。

新一代数学出现, 必然出现一套全新的数学符 号。研究静态问题的第一代数学, 其代表性符号是 阿拉伯数字、四则运算符、几何图形、英文字母和 矩阵等。研究动态问题的第二代数学, 其代表性符 号是极限、无穷大、无穷小、微分、积分、矢量和 形形色色的空间符号等。

我们不知道研究智慧问题的第三代数学, 其代 表性符号将是什么。本文建议, 在网络数学中, 可 使用互联网中常用的符号, 例如“@”和图 6 所示的 “二维码” 等。 


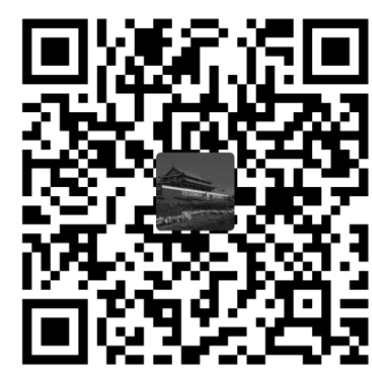

图 6. 表示文字数值信息的 “二维码”。

根据网络数学研究的对象和数学符号变化规 律, 我们给出网络数学的定义:

定义 3．研究互联网世界中各种信息的数量关系和 动态混沌空间，并用互联网中主要符号加以表达的 数学, 称为网络数学。

正如第二代数学大量使用第一代数学提供的工 具但与第一代数学有本质区别一样, 第三代数学也 必须能够使用第一代数学和第二代数学的工具但与 它们有本质区别。网络数学作为智能数学的一部分, 必须能够使用传统数学的工具, 但却不能是传统数 学简单拓展, 须有自己的内核。

之所以网络数学有可能成为智能数学的一部 分, 是因为互联网中以信息为载体, 流动着大量的 人类智慧。这些智慧在互联网中的累积、交溶、嬗 变和服务, 形态万千, 绝非个体人脑中的智慧形态。 网络数学哪怕触碰到互联网世界中人类智慧的一小 部分，其成就也会远在前述提及的人工智能之上。

一旦人们有了网络数学的帮助, 就能设计出挖 掘人类智慧的智联网界面，就能设计出集小智慧为 大智慧的数学模型来, 智联网就能被大规模地应用, 智联网也必将成为 “互联网+风险分析” 的主流。

就目前的各种数学理论而言, 因素空间理论有 可能为网络数学的形成提供帮助。

\section{5. 基于因素空间理论的网络数学框架}

因素空间的最初思想, 是汪培庄在解释随机性 的根源及概率规律的数学实质时产生的。1982 年在 文献[16]中给出了一个传统数学意义上的定义。

“因素” 是一个泛泛的概念, 既指构成事物本 质的成分，也指决定事物成败的原因或条件，又称 因子。生物学中的 “基因”，最初就被称为 “因素”。 在科学试验中, 影响试验指标的要素或原因, 称为 因素。
在因素空间理论中，因素的要意被数学符号替 代, 因素被数学地定义为一种映射。它把一个对象 $f$ 映射成为一个属性值 $X_{f}$ 。例如, 身高是一个映射, 它把一个对象 “张三” 映射成类似 “很高” 这样的 属性。这里, “身高” 就是全面描述人的 “因素”之 一。满足一定数学结构的, 由诸因素组成的一个集 合族，就称为一个因素空间。形式化定义如下：

定义 4. 令 $\left\{X_{f}\right\}(f \in L)$ 是一个因素集合族。如果 $L$ 是一个布尔代数, $L=(L, \vee, \wedge, c, 0,1)$, 且满足下列条 件，则称此集合族为一个因素空间，记为 $\left(X_{f}, f \in L\right)$ 。

(1) $X_{0}=\{\phi\}$;

（2）若 $L$ 的子集 $T$ 独立（即, $\forall \alpha, \beta \in T$, 当 $\alpha \neq \beta$, 必 $\alpha \wedge \beta=0$ ), 则

$$
X_{\gamma}=\prod_{\alpha \in T} X_{\alpha}
$$

这里 $\gamma=\vee\{\alpha \mid \alpha \in T\}, 0$ 和 1 分别是 $L$ 中的最小和最 大元素， П表示笛卡尔乘积。

虽然在过去的 30 多年中，因素空间的研究，一 直在传统数学的框架内, 并且以代数系统为主, 但 正如模糊集、粗糙集在传统数学的基础上拓展了人 们的视野一样，因素空间理论提供了从 “因素” 看 问题的视角。有了这种视角, 在一个以模糊概念构 成的状态空间中，由信息扩散技术处理观测样本生 成的原始信息矩阵, 可以转化为因果型模糊关系 ${ }^{[17]}$ 。 尽管因素空间理论处理实际问题的能力仍停留在传 统数学可以触及的问题上，但这并不意味着因素空 间理论不具有超越传统数学的发展空间。关键是因 素空间理论和方法的研究人员能否挣脱传统思维惯 性的束缚, 放弃诸如 “因素库”, “知识挖掘算法”, “定性相空间” 等的研究, 重点放在传统数学难以 发挥作用的问题上, 展开全新的研究。建设网络数 学框架, 或许是因素空间理论全新发展的第一步。

中国传统数学的基本框架, 由方田、粟米、差 分、少广、商功、均输、盈不足、方程、旁要等构 成, 包括四则运算法则等。它们在《九章算术》中 有详细介绍。西式的现代数学, 其基本框架由代数、 几何和微积分等构成。概率论, 也是由这个框架中 的元素构成。集合论, 是这个框架的 “地基”。动一 动地基, 框架就伸出去一大块。模糊数学就是这么 
来是。网络数学, 不可能由这个框架中的元素构成, 因为集合论的 “映射” 决定了传统数学只能处理结 构化信息(Structured Information)，而在网络中流动 的代表人类智慧的很多信息，是非结构化的，它们 不能按预先定义的方式进入网络。现有用传统数学 研制的语义分析工具，对非结构化的柔性信息无能 为力, 但人脑却能处理这类信息。例如, “洪水齐腰 深”, 没有明确定义, 不能形式化, 用模糊集表达, 也难尽人意, 因为个头高矮的人, 齐腰深的深度并 不一样。

基于因素空间理论的网络数学框架, 首要目标 是能处理柔性信息, 这就注定了不能在传统数学的 框架中打圈圈, 首先要从数学符号上有所突破, 更 是要设法离开代数系统。为此, 我们先定义网络数 学的符号如下:

定义 5. 设@是一个在互联网中通用的符号, 称它是 一个网络数学的符号。

例如, 微信中使用的图 7 中一些有代表性的符 号, 可选作网络数学的符号。图 6 中的“二维码”, 也可作网络数学符号的一员。

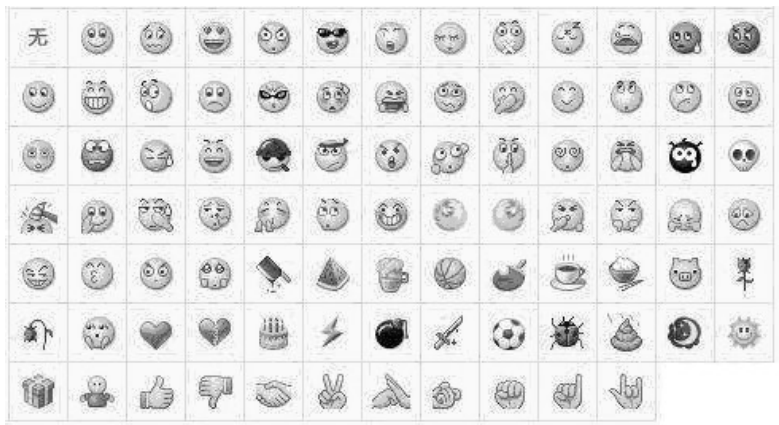

图 7. 微信中使用的符号。

我们将网络数学中使用的所有符号组成的集合 记为 $\Omega$, 其内的元素记为@。网络数学中的结构定义 如下:

定义 6. 设 $\omega$ 是 $\Omega$ 的一个非空子集，一个能在互联网 中描述 $\omega$ 的某种群体行为的方式, 称为一个结构, 记 为 $\S(\omega)$ 。

例如, 以网络数学符号表征因素的一个因素藤, 或许能成为网络数学中的一个结构 (图 8)。

因素空间与信息科学的相结合, 正在为揭示人 类对客观世界认知的原理, 为表达知识, 开展网络 背景下的人工智能研究, 提供新的数学工具。因素

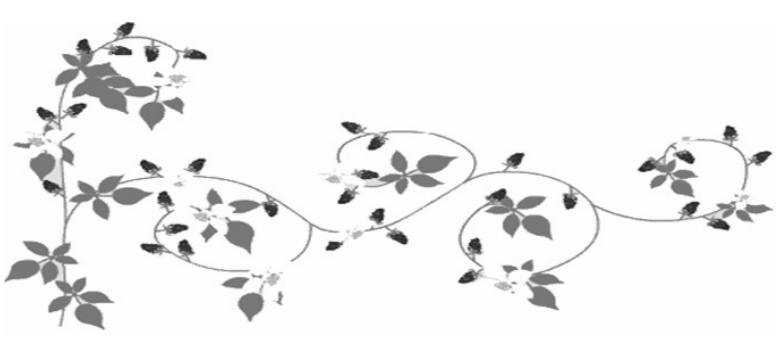

图 8. 因素藤结构。

空间架构体系在智联网上的实现 ${ }^{[9]}$, 或许意示着因素 空间理论已经开始走向网络数学。

\section{6. 网络数学支持的智联网}

正如现在的计算机仿真用面向对象技术进行模 块化搭建, 不需在底层编程一样, 网络化数学将为 人们提供面向智慧的技术，构造智联网不再需要懂 得网络语言, 而是用一些模块搭建。有了这些工具, 构造智联网就会变得简单，使用智联网就会便捷。

当因素空间成为网络数学的一部分时, 其功能 之一，是用多种形态的因素空间来承担分解和溶合 多个智能体所提供信息中蕴藏的智慧成分, 并将小 智慧提升为大智慧, 努力达到 “三个臭皮匠顶个诸 葛亮”的作用。

不失一般性，我们用图 9 来代表三种基本的因 素空间。图 9（a）中的 “藤式因素空间” 用于表达 错综复杂的因素关系; 图 9（b）的 “动作因素空间” 用于表达复杂而有规律 (含 “随机规律”) 的变化事 物; 图 9（c）的 “趋势因素空间” 用于表达因素发 表动态。

正如传统数学的符号举不胜举, 但大多是一些 基本符号的变体一样, 描述主客观世界的因素空间 符号，应该很多，但也会有一些是基本的。

用图 9 的因素空间符号 $\aleph, \mathfrak{I}, \mathfrak{R}$ 加上“@”和 “二 维码”, 可构造一个简单的, 由网络数学支持的智联 网模型。

针对一个具体的问题，我们用 $@_{1}, @_{2}, \ldots$, $\Theta_{n}$ 代表 $n$ 个智能体提供的信息、经验和判断。每一 个@进入智联网, 就类似于一封电子邮件发给一位 综合分析大师一样，内含丰富的信息和智慧。

$@_{1}, @_{2}, \ldots, @_{n}$ 经由网络数学中的因素空间 进行分解、溶合, 集成智慧, 形成产品, 其输出用 二维码表示。对图 2 进行拓展, 得到一个网络数学 支持的简单智联网，如图 10 所示。 


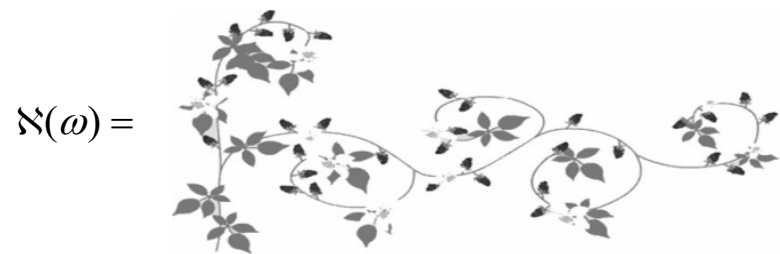

（a）藤式因素空间

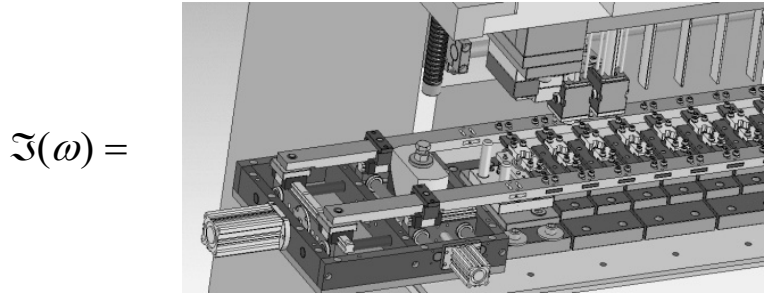

（b）动作因素空间

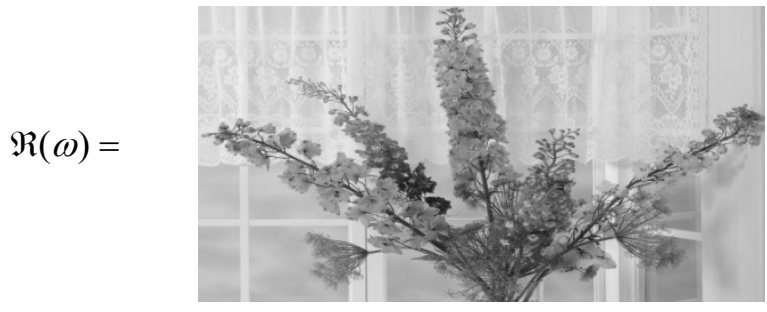

（c）趋势因素空间

图 9. 网络数学中三种基本的因素空间。

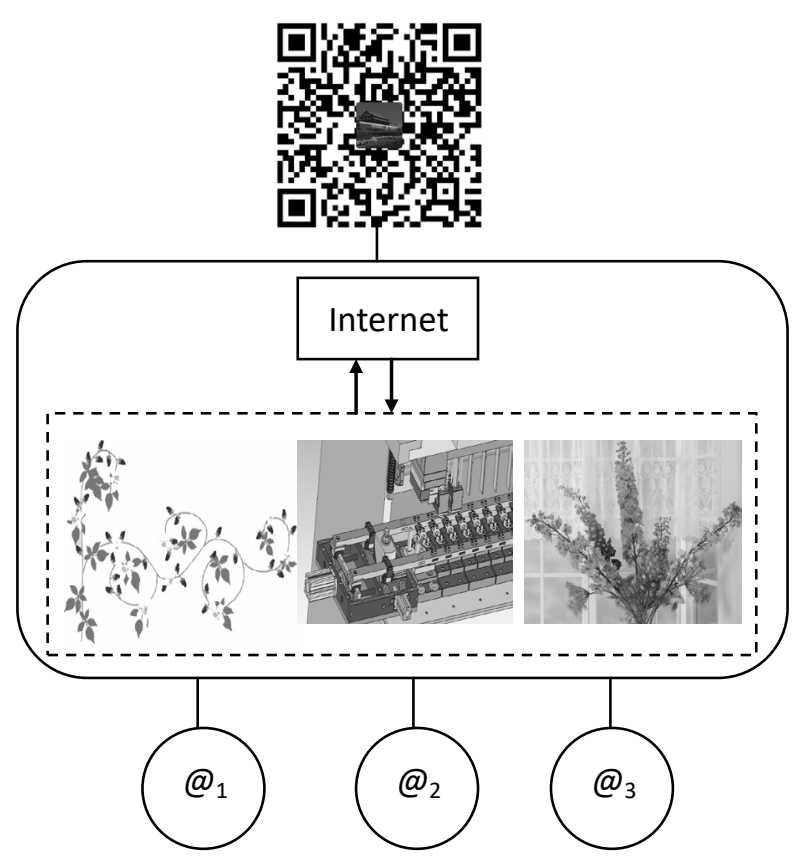

图 10. 用因素空间分解、溶合并提升智慧的智联网

\section{7. 结论和讨论}

智能化风险分析的工作，并非用传统意义上的 人工智能进行，更不是天罗地网的监测系统的自动 化分析，而是依托互联网平台，以集小智慧为大智 慧为准则的智能化工作。因为只有人类才具有 “用 有限的知识, 认识无限的世界” 的动机, 才有对 “与 不利事件有关的未来情景-一风险” 展开分析的能力。

今天, 商业化的人工智能, 是自动化意义下的 “灵巧” (Smart) 系统, 并非能创造知识的智能, 不具有 “智慧” (Intelligence)。

发端于在线风险分析服务的智联网, 追求的是 依托互联网, 发挥利益相关者的群体智慧, 对具体 的风险进行跟踪、识别、判断和管理, 使 “互联网+ 风险分析” 成为可能。

目前研制出来智联网, 受传统数学的限制, 只 具有共享信息、实时在线分析的能力, 其核心技术 是信息扩散技术。为了提高智联网的能力，须发展 智能数学。网络数学是实质性探索智能数学的开始。 因素空间理论有可能为网络数学的形成提供帮助。

因素空间在智联网中或许能承担分解和溶合多 个智能体所提供信息中蕴藏的智慧成分，并将小智 慧提升为大智慧的任务。

一旦在智能数学的支持下, 智联网具有了 “三 个臭皮匠顶个诸葛亮” 的能力, “互联网+风险分析” 就会成为人们进行风险分析的首选。

\section{参考文献}

[1] Huang C F. Internet of intelligences in risk analysis for online services. Journal of Risk Analysis and Crisis Response, 2011, 1(2): 110-117.

[2] 北京崇安智联科技服务有限责任公司．高考志愿填报 智联网服务平台一联接智慧,网获知识 (available at: http://www.cazl.cn/ioiserver/index.php?r=site/main), 2013.

[3] 艾福利．自然灾害风险分析智联网服务平台构建与应 用研究. 北京师范大学博士学位论文,2014.

[4] 北京崇安智联科技服务有限责任公司. 风险学会 “优 秀论文奖” 评选系统(available at: http://www.cazl.cn/ risktech/yxlw/),2014.

[5] 曾凡雷. 属性拼图智联网风险分析方法及其服务平台 的构建与应用研究. 北京师范大学博士学位论文, 2016.

[6] Huang C F, Wu T, Renn O. A risk radar driven by internet of intelligences serving for emergency management in community. Environmental Research, 2016, 148: 550-559.

[7] 苏妩. 信息收集去中心化在风险评估中的应用---海洋 环境风险管理智联网平台研究. 北京师范大学硕士学 位论文,2016. 
[8］王蔚丹. 地震宏观异常的综合度量及其在智联网服务 平台上的应用研究. 北京师范大学博士学位论 文,2017.

[9] 郭君. 自然灾害概率风险时效性评价的因素空间架构 体系及其在智联网上的实现. 北京师范大学博士学位 论文, 2017 .

[10] 梁屹. 网络互动模式下洪水灾害风险沟通共识获得的 方法一基于智联网途径. 北京师范大学博士学位论 文,2017.

[11] 刘锋,彭㥷,刘颖. 从人脑的结构机理看互联网的进化. 人类工效学, 2009,15(1): 11-14.

[12]Heylighen F. Conceptions of a Global Brain: an historical review, in Evolution: Cosmic, Biological, and Social, eds. Grinin L E, Carneiro R L, Korotayev A V and Spier
F. (Uchitel Publishing, Volgograd, Russia, 2011) pp.274-289.

[13] Huang C F. Multiple Internet of Intelligences for Risk Analysis. Journal of Risk Analysis and Crisis Response, 2014, 4(2): 61-71.

[14] Steinbeis Advanced Risk Technologies. iNTeg-Risk RiskTicker(available at: http://www.integrisk.eu-vri.eu/ \#bl2099),2013.

[15] 刘云丰,陈洪,刘忠,史光亚. 智能数学:内容、方法及意 义. 自然杂志, 1987,(4):255-262.

[16] 汪培庄,Sugeno M. 因素场与模糊集的背景结构. 模 糊数学, 1982,(2):45-54.

[17] 黄崇福,王家鼎. 模糊信息优化处理技术及其应用. 北京:北京航空航天大学出版社,1995. 